\title{
Coherence resonance and noise-induced synchronization in globally coupled Hodgkin-Huxley neurons
}

\author{
Yuqing Wang, David T. W. Chik, and Z. D. Wang \\ Department of Physics, The University of Hong Kong, Pokfulam Road, Hong Kong, People's Republic of China
}

(Received 5 August 1999; revised manuscript received 27 September 1999)

\begin{abstract}
The coherence resonance (CR) of globally coupled Hodgkin-Huxley neurons is studied. When the neurons are set in the subthreshold regime near the firing threshold, the additive noise induces limit cycles. The coherence of the system is optimized by the noise. The coupling of the network can enhance CR in two different ways. In particular, when the coupling is strong enough, the synchronization of the system is induced and optimized by the noise. This synchronization leads to a high and wide plateau in the local CR curve. A bell-shaped curve is found for the peak height of power spectra of the spike train, being significantly different from a monotonic behavior for the single neuron. The local-noise-induced limit cycle can evolve to a refined spatiotemporal order through the dynamical optimization among the autonomous oscillation of an individual neuron, the coupling of the network, and the local noise.

PACS number(s): 87.18.Sn, 87.19.La, 05.40.-a
\end{abstract}

The phenomenon of stochastic resonance (SR) has been intensively studied for the last decade [1]. The response of a noisy nonlinear system to a deterministic signal can be optimized by noise. Recently, it has been shown that, in the absence of a deterministic signal, the noisy nonlinear system exhibits SR-like behavior [2-8]. This phenomenon, which is referred to as coherence resonance (CR) or autonomous SR, was first discussed in a simple autonomous system in the vicinity of the saddle-node bifurcation [2,3]. The nonuniform noise-induced limit cycle leads to a peak at a definite frequency in the power spectrum. The signal-to-noise ratio (SNR) increases first to a maximum and then decreases when the intensity of noise increases, showing the optimization of the coherent limit cycle to the noise. The frequency was observed to shift to a higher value by increasing the noise intensity. The CR has also been found in excitable systems, e.g., the Fitz Hugh-Nagumo model [4], the Hodgkin-Huxley (HH) model [5], the Plant model, and the Hindermarsh-Rose model [6]. Moreover, an experimental evidence of CR was reported very recently [8].

Synchronization and SR in the coupled nonlinear stochastic systems have also attracted growing interests in recent years [9-15]. Regardless of whether the system is locally or globally coupled, the coupling can enhance the signal transduction and the SNR of the local unit. The coupling strength can be considered to be another tuning parameter of SR. Meanwhile, the noise-induced global synchronization, which coincides with the optimized local performance of the single element in the network, is observed. Moreover, Kurrer and Schulten [16] have studied analytically a model of globally coupled stochastic neurons and found noise-enhanced synchronization. On the other hand, Rappel and Karma [17] studied properties of the power spectra of globally coupled neurons and found a new effect of noise-induced $\delta$-peak. Recently, the synchronization and the effect of CR in two coupled excitable oscillators are also investigated numerically and experimentally [18].

In this paper, the $\mathrm{CR}$ of the globally coupled $\mathrm{HH}$ neurons is studied numerically. We show that the coupling of the network can enhance CR in two different ways. When the coupling is weak, the CR phenomenon behaves similarly to that of a single neuron, and no spatiotemporal order can be observed. When the coupling becomes strong enough, the local measure of coherence jumps up to a wide plateau first and then jumps down from the plateau as the intensity of noise increases, due to the spatiotemporal synchronization of the network. The coupling tends to stabilize the noiseinduced limit cycle and synchronization. The peak frequency of noise-induced limit cycle is selected to be the spatiotemporal order through the optimization among the excitability of a single neuron, the coupling of the network, and the local noise. The phase of synchronized oscillation is also determined through the dynamical evolution of the system. Because the $\mathrm{HH}$ model serves as a paradigm for spiking neurons, we may relate our results to the existence of coherent spontaneous oscillations observed in the brain cortex [19-21].

A network of coupled $\mathrm{HH}$ neurons is described by the following equations:

$$
\begin{aligned}
\frac{d V_{i}}{d t}=f_{i}\left(V_{i}, m_{i}, n_{i}, h_{i}\right) & -I_{i}(t)-\eta_{i}-\frac{1}{N-1} \sum_{j=1, j \neq i}^{N} J_{i j} S_{j}, \\
\frac{d m_{i}}{d t} & =\frac{m_{\infty}(V)-m_{i}}{\tau_{m}(V)} \\
\frac{d n_{i}}{d t} & =\frac{n_{\infty}(V)-n_{i}}{\tau_{n}(V)} \\
\frac{d h_{i}}{d t} & =\frac{h_{\infty}(V)-h_{i}}{\tau_{h}(V)},
\end{aligned}
$$

where $f_{i}\left(V_{i}, m_{i}, n_{i}, h_{i}\right)$ is

$$
\begin{aligned}
f_{i}\left(V_{i}, m_{i}, n_{i}, h_{I}\right)= & -g_{N a} m_{i}^{3} h_{i}\left(V_{i}-V_{N a}\right)-g_{K} n_{i}^{4}\left(V_{i}-V_{K}\right) \\
& -g_{L}\left(V_{i}-V_{L}\right) .
\end{aligned}
$$


Each neuron is described by a set of four time-dependent variables $\left(V_{i}, m_{i}, n_{i}, h_{i}\right)$, where $V_{i}$ is the membrane potential, $m_{i}$ and $h_{i}$ the activation and inactivation variables of sodium current, and $n_{i}$ the activation variable of potassium current. The meaning and detailed values of the parameters can be found in Ref. [22]. The simulation was done by using the fourth order Runge-Kutta method with the time step being taken as $0.01 \mathrm{msec}$.

Each neuron is subject to an independent noise $\eta_{i}$ with the same intensity, which is determined from an OrnsteinUhlenbeck process $\tau_{c} d \eta_{i} / d t=-\eta_{i}+\sqrt{2 D} \xi$, where $\xi$ is the Gaussian white noise [23]. $D$ and $\tau_{c}(=0.1 \mathrm{msec})$. are the intensity and the correlation time of the noise, respectively. $I_{i}(t)$ is the input current, which will be time-independent and will bias the neuron near the saddle-node bifurcation. The last term in Eq. (1) is the coupling of the network. The effect of the firing activity of $j$ th neuron on the $i$ th neuron is modeled by an impulse current to the $i$ th neuron, which is proportional to the efficacy of the synapse $J_{i j}$ and is generated when the $j$ th neuron is active. $J_{i j}=J$ for all pairs of neurons with $J$ the coupling strength of the system. The neuron is active whenever its membrane potential exceeds a threshold $V^{*}\left(=0 \mathrm{mV}\right.$ here). This activity can be denoted by $S_{j}$ $=\Theta\left(V_{j}-V^{*}\right)$, where $\Theta(x)=1$ if $x \geqslant 0$ and $\Theta(x)=0$ if $x$ $<0$. In the present simulation, only the excitatory coupling is considered $(J>0)$; that is, the last term is the excitatory postsynaptic potential (EPSP) received by the single neuron.

The HH neuron is an excitable one. For a dc input current $I_{0}$, the firing threshold is $I_{c}=6.2 \mu \mathrm{A} / \mathrm{cm}^{2}$. The spike limit cycle occurs at $I_{c}$ due to the saddle-node bifurcation. To observe the CR, we set the input current $I_{0}=6.0 \mu \mathrm{A} / \mathrm{cm}^{2}$ for each neuron [24]; that is, the system is set in the subthreshold regime near the threshold or saddle-node bifurcation. For one single $\mathrm{HH}$ neuron, the coherence resonance was discussed in detail in Ref. [5]. In the present simulation, we focus on a globally coupled network, and attempt to extract more significant information of CR.

The CR exhibits two different behaviors when the coupling intensity changes. They can be seen in the power spectrum of the output spike trains. In the absence of noise, a single neuron stays at the quiescent state in which the membrane potential is below $V^{*}$. In this case, there would be no synaptic transmission between the neurons, and the whole network would stay at the quiescent state. If an independent local noise $(D \geqslant 0.3)$ is applied to each neuron, the system begins to fire spike trains. When the coupling is weak (e.g., $J=5.0$ ), the power spectrum densities of the spike trains for different intensities of noise are shown in Fig. 1(a). A broad peak can be seen, similar to the single neuron case (see Fig. 2 in Ref. [5]). This behavior of CR is similar but different to that of a single neuron.

When the coupling of the network is strong (e.g., $J$ $=10.0$ ), the power spectrum densities of the spike trains for different intensities of noise are shown in Fig. 1(b). As the noise is weak, a broad peak is also observed. However, when the noise intensity increases, the peak becomes higher and sharper. This type of power spectrum is quite different from that for usual CR discussed previously. The sharp peak is induced by the network itself and locked at the frequency of spontaneous limit cycle. The detail of this kind of power spectrum has been addressed in Ref. [17]. When the noise (a)

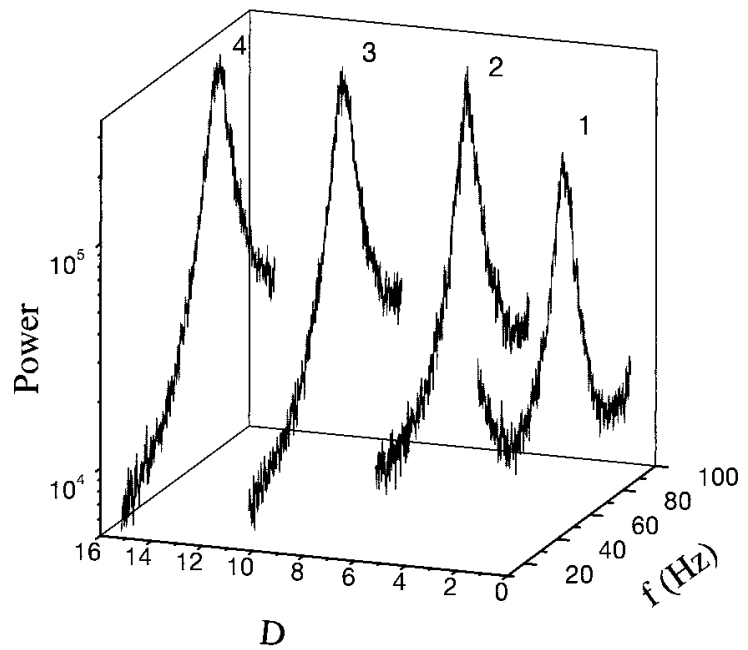

(b)

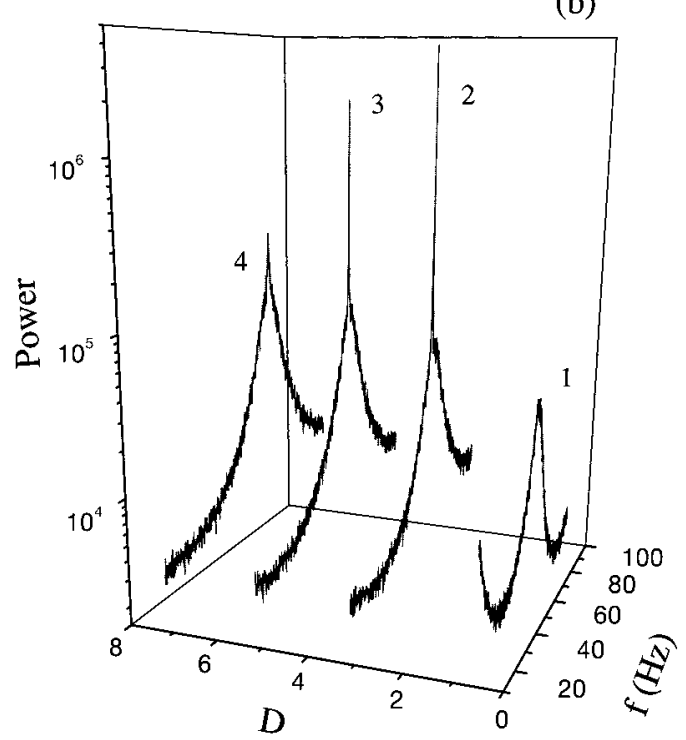

FIG. 1. (a) Power spectrum of the spike trains with a weak coupling strength $J=5.0$ for the noise intensity $D=1.0,5.0,10.0$, and 15.0. (b) The power spectrum of the spike train with a strong coupling $J=10.0$ for $D=0.5,3.0,5.0$, and 7.0. The size of the network $N=1000$.

intensity increases further, the sharp peak tends to become broad, keeping the general trend of CR in the single neuron case.

The difference of spatiotemporal orders of the network leads to such two different behaviors of CR. In previous studies of the conventional SR, each unit in the network receives a common external signal with the same frequency and phase. The external signal represents an external "clock" leading to the synchronization of the whole system. So the tuning of the synchronization to the local noise, which coincides with the local SNR behavior, can be observed when the external signal is sufficient strong [9]. However, in the case of CR, the situation is different. There is no such kind of global tuning in the network. The local oscillation of each unit is noise-induced limit cycle. The phase is random in time and is irrelevant to each other. Besides, a broad peak in Fig. 1(a) means that the frequency has some uncertainty. As a result, the synchronization is not guaranteed in the case of $\mathrm{CR}$. 

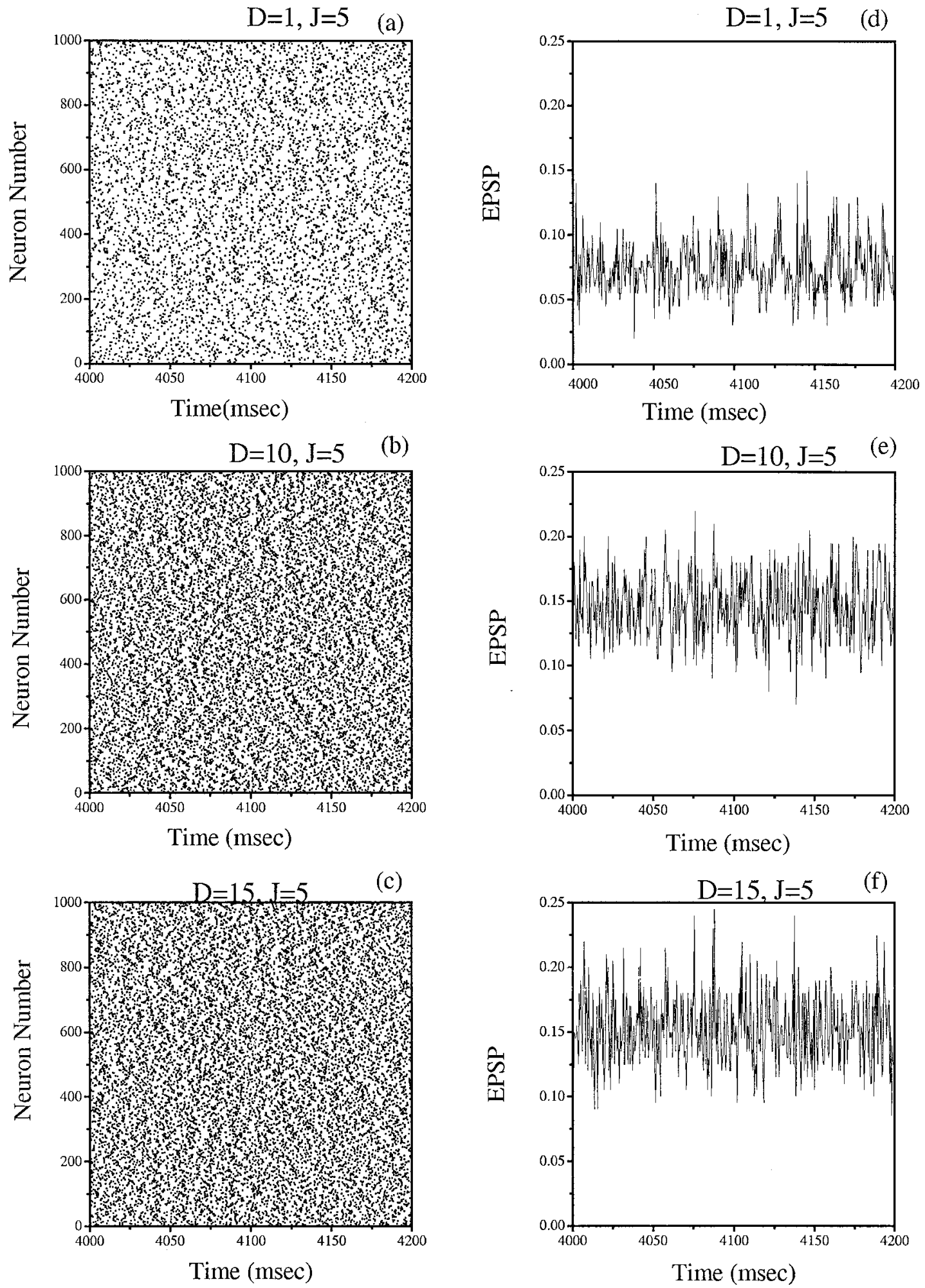

FIG. 2. Raster of the network and corresponding excitatory postsynaptic potential (EPSP) of a neuron with $J=5.0$ for different intensities of noise: $D=1.0[(\mathrm{a})$ and (d)], $D=10.0[(\mathrm{~b})$ and (e)], and $D=15.0[(\mathrm{c})$ and (f)]. The network size $N=1000$.

When the coupling is weak, the raster records all the firing events in the network, and the corresponding EPSP of a single neuron for different intensities of noise are shown in Fig. 2. From Figs. 2(a)-2(c), we can see that there is no synchronization in the system. Especially, Fig. 2(b) appears to be the most coherent state $[D=10.0$, shown in Fig. 4(a) later]. To see the influence of the network on the local unit, the EPSP of an arbitrarily chosen neuron is shown in Figs. 2(d)-2(f). There is a tendency that the EPSP increases when the intensity of noise increases. The power spectrum of the EPSP has a broad peak, which coincides with the CR frequency, similar to that of the spike train (not shown here).

Figure 3 illustrates how the synchronization can be observed when the coupling is strong. It is shown in the raster [Figs. 3(a)-(c)] that, when the noise is weak $(D=0.5)$, there is no synchronization. Its corresponding power spectrum is 

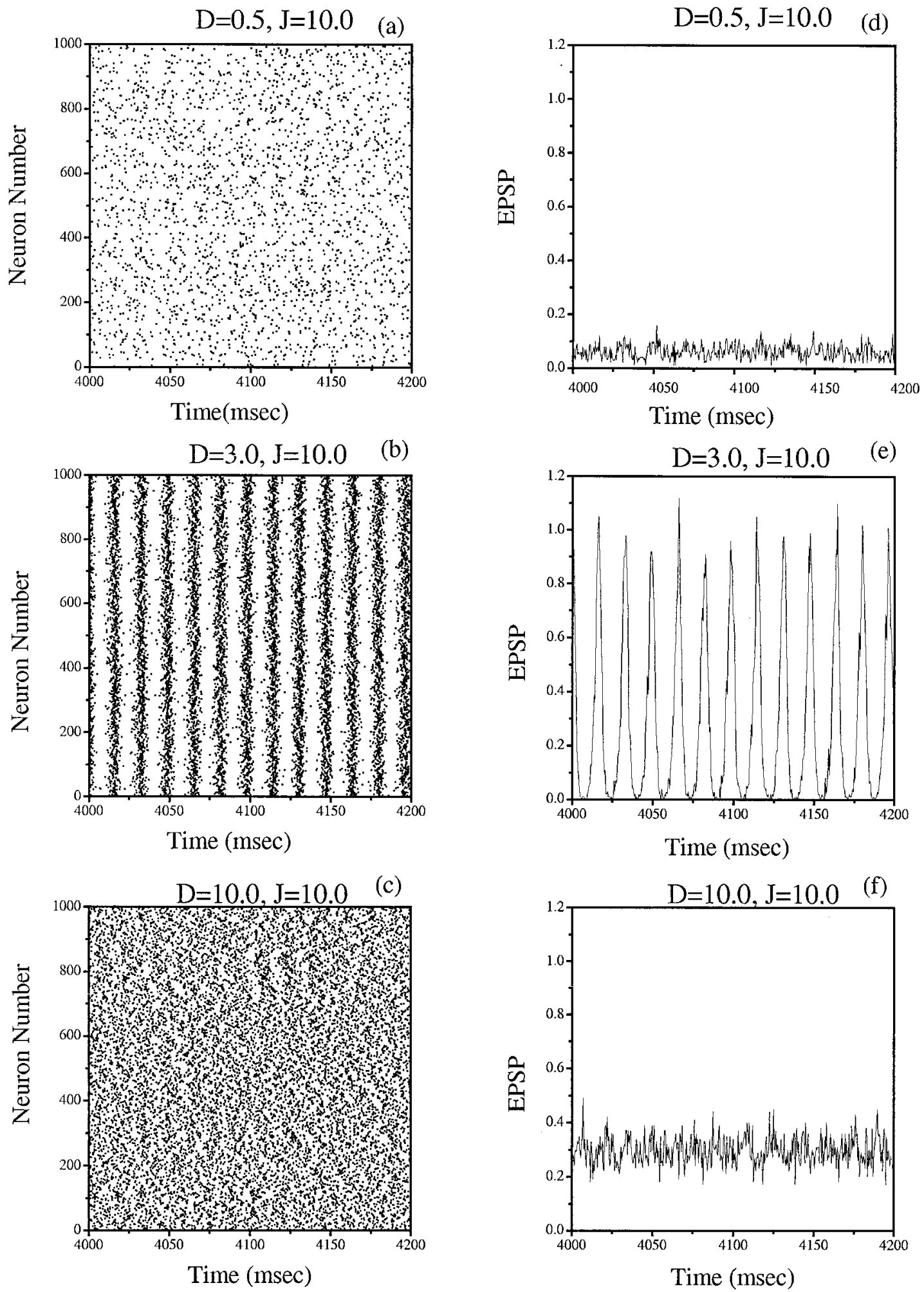

FIG. 3. Raster of the network and corresponding excitatory postsynaptic potential (EPSP) of a neuron with $J=10.0$ for different intensities of noise: $D=0.5$ [(a) and (d)], $D=3.0[$ (b) and (e)], and $D=10.0$ [(c) and (f)]. The network size $N=1000$.

given in line 1 in Fig. 1(b). When the noise intensity increases, as shown in Fig. 3(b), the synchronization can be observed. Note that this spatiotemporal order is achieved by increasing the intensity of the independent local noise in the absence of external periodic forcing. As shown in Fig. 3(e), the EPSP received by a single neuron has an explicit periodicity, that is, the network produces a kind of periodic oscil- lation due to the synchronization, which is quite similar to a deterministic signal input to each neuron. The corresponding power spectrum density of the spike train is shown as line 2 in Fig. 1(b). The sharp peak comes from the periodic EPSP, which reflects the effect of the synchronization on the local unit, in agreement with the work on the coupled integrate and fire neurons [17]. When the noise intensity increases 
further, the synchronization is destroyed; both the explicit periodicity of the EPSP and the high peak in the power spectrum of the spike train disappear.

Physically, the spatiotemporal order is established through the dynamical evolution of the system. As shown in Eq. (1), the EPSP that each neuron receives is the average of the events of the other $N-1$ neurons. Even if there is no synchronization in the system, the power spectrum of the resulted EPSP should have a dominant frequency of the limit cycle. This noise-induced EPSP is aperiodic. Its intensity and quality are dependent on the intensity of noise and the coupling strength. When the coupling strength is weak, the EPSP is very small in comparison with the intensity of the local noise. No correlation between the output spike train and the input EPSP can be established. When the coupling strength is strong enough, the situation will be different. Although the EPSP is still too small for a weak noise, the quality of EPSP is improved and the intensity is increased as the noise increases, due to the CR in the single element level. Since the input current contains a signal with the same frequency as the output, the output as well as the EPSP will be refined. This is a process of positive feedback. Because the EPSP is the average output of other neurons, the local neuron tends to keep the pace of such an averaged signal through the dynamical optimization process. Finally, a spatiotemporal order can be reached and the frequency of oscillation, which is just the frequency of CR, is "selected" by the dynamical process. If the noise intensity increases further, the synchronization is destroyed. So the EPSP can be viewed as a kind of indirect feedback. The EPSP is noise-induced and can be optimized by noise, while such local noise disturbs the feedback by adding irregularity at each time step. On the other hand, when the coupling is significant, the positive feedback is established. As a result, the EPSP will evolve gradually to become an identical periodic forcing on every single element in the system. The synchronization can be observed and optimized by the noise. Due to the feature of CR in the globally coupled neurons, regardless of whether the system is in the synchronized or desynchronized state, the frequency locking at the $\mathrm{CR}$ frequency always exists. The synchronization shown in Fig. 3(b) is a kind of phase locking of all the elements in the network.

Such noise-induced synchronization possesses two interesting features. First, the synchronization frequency is dependent on the local noise and the coupling. Second, the phase of spatiotemporal oscillation is determined by the dynamical evolution of the system itself. Because of this, the peak frequency of CR is locked at the frequency of the synchronized oscillation. However, the phase of the synchronized oscillation is "selected" by the indirect feedback process which is sensitive to the detail process in the noisy environment. For example, different initial conditions of the simulation lead to the same frequency but different phases of the synchronized oscillation.

We can characterize CR quantitatively via a coherence factor $\beta$ [2], which is the measure of coherence and defined as

$$
\beta=h\left(\Delta \omega / \omega_{p}\right)^{-1},
$$

where $h$ and $\omega_{p}$ are the height and the frequency of the peak, and $\Delta \omega$ is the width of the peak at the height $h_{1}=e^{-(1 / 2)} h$.
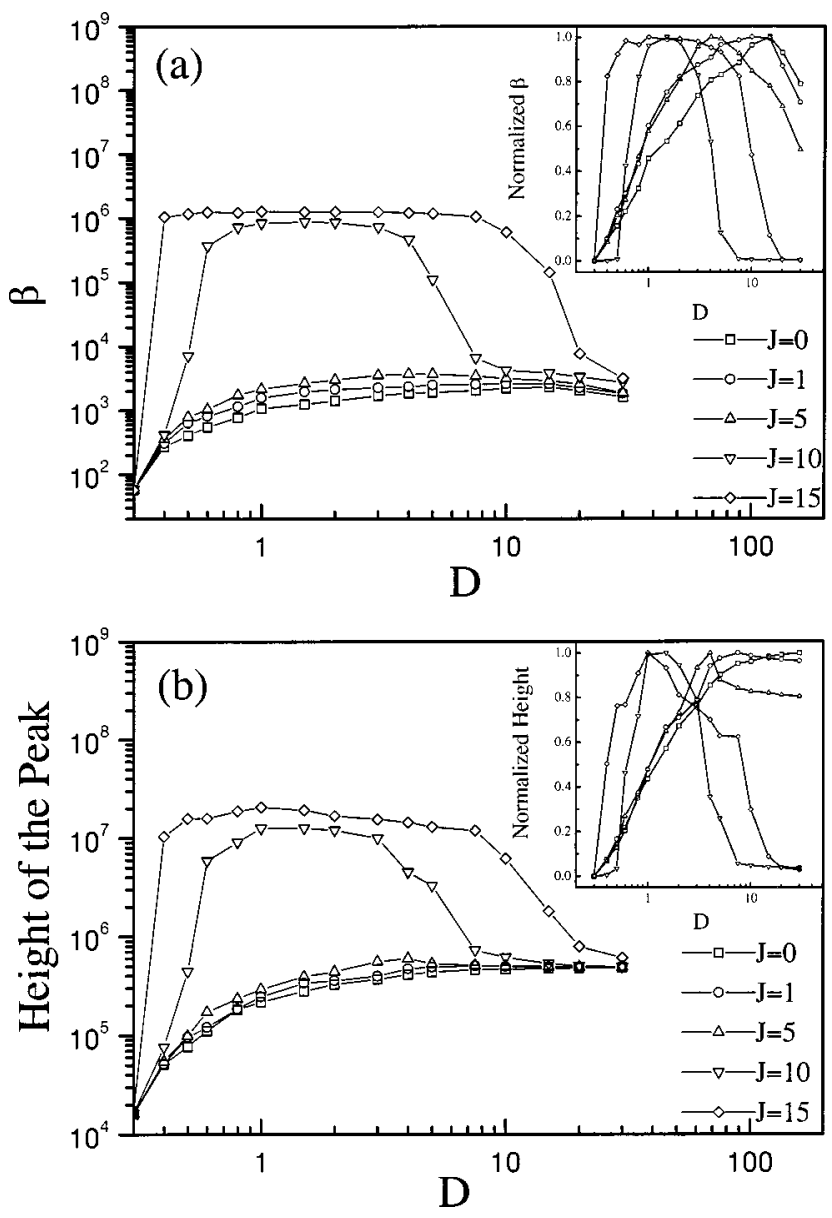

FIG. 4. (a) The measure of coherence $\beta$ vs the intensity of noise for different coupling strengths. Inset: The normalized coherence factor $\beta$ versus the intensity of noise. (b) The height of the peak of the power spectrum vs the intensity of noise for different coupling strengths. Inset: The normalized peak height versus the intensity of noise. The size of the network is $N=100$. The lowest lines in (a) and (b) are the same for the single neuron case.

The $\beta$ versus the noise intensity $D$ for different couplings of the network is shown in Fig. 4(a). When $D$ increases, $\beta$ increases first and then decreases after reaching the maximum. The coupling may be viewed as a tuning parameter of CR. For comparison, the $\mathrm{CR}$ of a single neuron case is also displayed in the figure $(J=0)$. The enhancement of $C R$ is significant when the coupling is stronger. When the coupling is weak, there is no spatiotemporal order in the system. The value of $\beta$ is the same order of the magnitude as that of the single neuron case, and similar $\beta-D$ curves are exhibited in the two cases. However, when the coupling becomes strong enough, the $\beta$ increases dramatically with $D$ at first, showing the onset of synchronization, and then a wide plateau is followed, indicating that the self-evolved spatiotemporal order is stable against a large range intensity of local noise. The normalized $\beta$ vs the noise intensity for different couplings is also shown in the inset of Fig. 4(a) .

The difference of the $\mathrm{CR}$ in the single neuron case and the coupled neurons can be seen in Fig. 4(b), in which the peak height of the power spectrum densities of the spike train is plotted against the noise intensity $D$ for different couplings of the network. In the single HH neuron case $(J=0)$, the height of the peak increases monotonically as the noise in- 
creases [see also Fig. 4(b) in Ref. [5]]. In the coupled HH neurons, similar to Fig. 4(a), a bell-shaped curve is observed. Once the synchronization is established, the peak height increases dramatically. On the other hand, even when the coupling is weak and no synchronization is established, as shown in the inset of Fig. 4(b), the bell-shaped curve can still be observed $[J=1$ and $J=5$ curves in Fig. 4(b)]. This means that the height of CR peak is tuned by the noise in the absence of synchronization. As shown in Figs. 2(d)-2(f), the EPSP can be regarded as a kind of aperiodic signal that has the same frequency as the output. The tuning to the noise of such an aperiodic signal is similar to SR; however, unlike the usual SR, the EPSP here is produced by the network itself through CR. The intensity and quality of the EPSP are different for different strengths of noise due to the effect of CR. The effect of CR can be enhanced significantly by the coupling, even when there is no synchronization.

Figure 5(a) illustrates how the $\beta$ changes with the size of the strongly coupled network $(J=10.0)$. Clearly, the $\beta-D$ curve changes little whenever the number of the neurons in the network is larger than 50, with the onset-point and the end-point of synchronization being almost unchanged. Although the network is globally coupled, the degree of synchronization is roughly irrelevant to the size of the network if it is sufficiently large.

Figure 5(b) shows the peak frequency of CR as a function of the intensity of noise for different coupling strengths. We can see that, regardless of the coupling strength, the frequency will increase when the noise increases, with the same tendency as that for a single neuron case. On the other hand, the frequency increases as the coupling strength increases, tuning CR in another way. Moreover, there is no dramatic change of the frequency when the spatiotemporal order is established. In fact, we cannot see the difference of synchronized and nonsynchronized states of the system from this kind of plot. Both are CR states.

Finally, we address the relevance of the CR of the globally coupled $\mathrm{HH}$ neurons to the activities of realistic neural systems. In recent years, synchronized spontaneous oscillations have been observed in the brain cortex and are proposed to possess a binding function, where the spatiallydistributed neurons resonate to generate large function states that bring about cognition [19-21]. From the simulations, we may elucidate how these synchronized spontaneous oscillations are established. It would be the CR state. The frequency of oscillation is determined by the excitability of a single neuron, the coupling of the network, and the noise. On
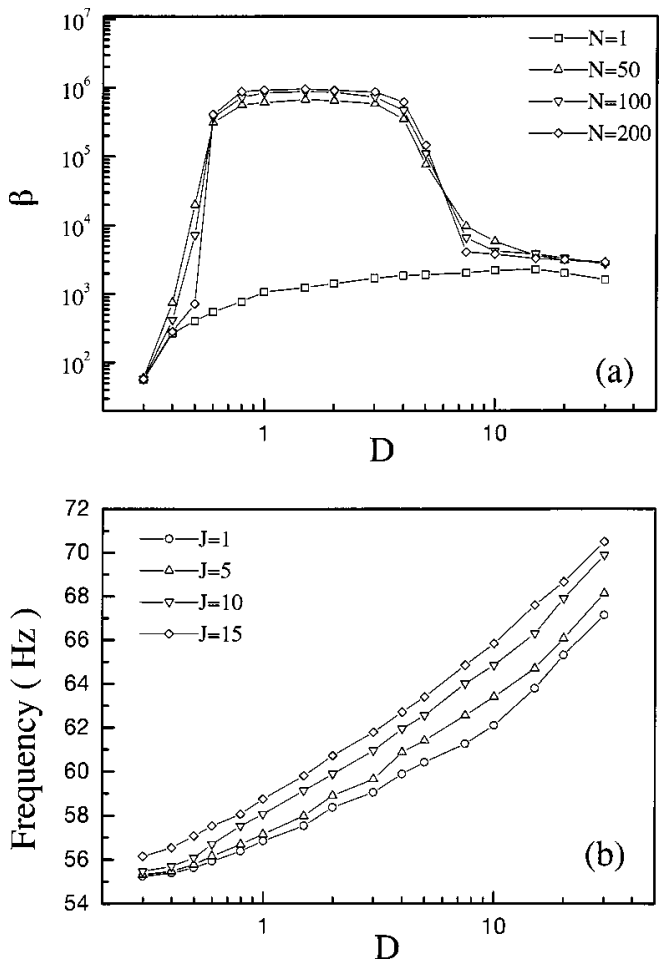

FIG. 5. (a) The measure of coherence $\beta$ vs the intensity of noise for different sizes of the network when $J=10.0$. (b) The frequency of $\mathrm{CR}$ vs the noise intensity for different coupling strengths. The size of network $N=100$.

the other hand, the synchronization may be noise-induced, giving a possibility that the noise would play an active role in neural activities. The synchronized state would be stable in a large range intensity of the local noise. This feature would enable the neural system to fulfill the cognition function in a noisy environment.

In summary, we have studied the CR of globally coupled network of HH neurons. It is found that, when the coupling is strong, the synchronization is induced and optimized by the noise. The frequency of CR of the local element is locked at the spatiotemporal oscillation frequency, and the phase of spatiotemporal oscillation is determined by the dynamical evolution. A wide plateau in the $\beta$ - $D$ curve was observed for the strongly coupled network with large sizes, indicating a stable spatiotemporal order in a large range intensity of local noise. The effect of CR can be enhanced greatly by the coupling regardless of the spatiotemporal order of the system. Our results may be relevant to the synchronized spontaneous oscillations observed in some realistic neural systems.
[1] K. Wiesenfeld and F. Moss, Nature (London) 373, 33 (1995); A.R. Bulsara and L. Gammaitoni, Phys. Today 49, 39 (1996); L. Gammaitoni, P. Hänggi, P. Jung, and F. Marchesoni, Rev. Mod. Phys. 70, 223 (1998); W. Wang, Y. Wang, and Z.D. Wang, Phys. Rev. E 57, R2527 (1998); Y. Wang, Z.D. Wang, and W. Wang, J. Phys. Soc. Jpn. 67, 3637 (1998).

[2] G. Hu, T. Ditzinger, C.N. Ning, and H. Haken, Phys. Rev. Lett. 71, 807 (1993).

[3] W.J. Rappel and S.H. Strogatz, Phys. Rev. E 50, 3249 (1994).

[4] A.S. Pikovsky and J. Kurths, Phys. Rev. Lett. 78, 775 (1997).
[5] S.G. Lee, A. Neiman, and S. Kim, Phys. Rev. E 57, 3292 (1998).

[6] A. Longtin, Phys. Rev. E 55, 868 (1997).

[7] A. Neiman, P. Saparin, and L. Stone, Phys. Rev. E 56, 270 (1997).

[8] D.E. Postnov, S.K. Han, T.G. Yim, and O.V. Sosnovtseva, Phys. Rev. E 59, R3791 (1999).

[9] A. Neiman, A. Silchenko, V. Anishchenko, and L. Schimansky-Geier, Phys. Rev. E 58, 7118 (1998).

[10] A. Neiman, L. Schimansky-Geier, F. Moss, B. Shulgin, and 
J.J. Collins, Phys. Rev. E 60, 284 (1999); B.V. Shulgin, A. Neiman, and V. Anishchenko, Phys. Rev. Lett. 75, 4157 (1995).

[11] A. Silchenko, T. Kapitaniak, and V. Anishchenko, Phys. Rev. E 59, 1593 (1999).

[12] J.F. Linder, B.K. Meadows, W.L. Ditto, M.E. Inchiosa, and A.R. Bulsara, Phys. Rev. Lett. 75, 3 (1995); Phys. Rev. E 53, 2081 (1996).

[13] M. Morillo, J. Gomez-Ordonez, and J.M. Casado, Phys. Rev. E 52, 316 (1995); J.M.G. Vilar and J.M. Rub', Phys. Rev. Lett. 78, 2886 (1997).

[14] W. Wang and Z.D. Wang, Phys. Rev. E 55, 7379 (1997); W. Wang, G. Chen, and Z.D. Wang, ibid. 56, 3728 (1997).

[15] P. Jung and G. Mayer-Kress, Phys. Rev. Lett. 74, 2130 (1995).

[16] C. Kurrer and K. Schulten, Phys. Rev. E 51, 6213 (1995).

[17] W.-J. Rappel and A. Karma, Phys. Rev. Lett. 77, 3256 (1996).

[18] S.K. Han, T.G. Yim, D.E. Postnov, and O.V. Sosnovtseva, Phys. Rev. Lett. 83, 1771 (1999).
[19] R. Llinás and U. Ribary, Proc. Natl. Acad. Sci. USA 88, 2078 (1993).

[20] M. Steriade, I. Timofeev, N. Dürmüller, and F. Grenier, J. Neurophysiol. 79, 483 (1998).

[21] M.A.L. Nicolelis, L.A. Baccala, R.C.S. Lin, and J.K. Chapin, Science 268, 1353 (1995).

[22] A.L. Hodgkin and A.F. Huxley, J. Physiol. (London) 117, 500 (1952); D. Hansel, G. Mato, and C. Meunier, Phys. Rev. E 48, 3470 (1993).

[23] P. V. E. McClintock and F. Moss, in Noise in Nonlinear Dynamical Systems, edited by F. Moss and P. V. E. McClintock (Cambridge University Press, Cambridge, England, 1989). Vol. 3, p. 243.

[24] It is not necessary to assume the same $I_{0}$ for every neuron in the network. The only requirement is to set the neurons near the threshold or saddle-node bifurcation. For a random distributed $I_{0}$, we will observe almost the same phenomenon reported later on. 